\title{
OsHLH61-OsbHLH96 influences rice defense to brown planthopper through regulating the pathogen-related genes
}

\author{
Meiling Wang ${ }^{1,2}$, Dongyong Yang ${ }^{1,2}$, Feilong $\mathrm{Ma}^{1,2}$, Mulan Zhu $^{3}$, Zhenying Shi ${ }^{1}$ and Xuexia Miao ${ }^{1 *}$ (D)
}

\begin{abstract}
Background: In plants, basic helix-loop-helix (bHLH) proteins form the largest transcription factor (TF) family. Among them, HLH proteins are a small group of atypical members that lack the basic domain, and form dimers with bHLH proteins. Although bHLH proteins have been proved to play important roles in plant development and physiology, the function of HLH proteins is rarely studied, not to mention in plant biotic resistance. Brown planthopper (BPH) is a kind of rice-specific insect that causes devastating yield losses each year.
\end{abstract}

Results: In this study, we identified OsHLH61 gene that encodes HLH protein. OsHLH61 gene could be highly induced by BPH infestation. Furthermore, Methyl Jasmonic acid (Me-JA) and cis-12-oxo- phytodienoic acid (OPDA) induced expression of OsHLH61, while SA repressed it. We knocked down expression of OsHLH61 by RNA interference (RNAi), the transgenic plants were susceptible to BPH infestation. RNA-seq analysis revealed that some pathogen-related (PR) genes in the Salicylic acid (SA) signaling pathway that mediate plant immunity were obviously down-regulated in the OsHLH61 RNAi plants. Meanwhile, yeast two-hybrid assay and bimolecular luciferase complementation (BiLC) analysis identified bHLH096 to be an interacting factor of OsHLH61. Also, some PR genes were down-regulated in the OsbHLH96 over expressing lines. Expression of OsbHLH96 was inhibited. Besides, OsbHLH96 might interact with Jasmonate Zim-Domain3 (OsJAZ3).

Conclusion: Altogether, we identified an OsHLH61-OsbHLH96 complex that might mediate defense to BPH through regulating PR genes. And OsHLH61-OsbHLH96 might be important in mediating SA and JA signaling crosstalk.

Keywords: Oryza sativa, bHLH, HLH, Transcription factor, Brown planthopper, PR gene

\section{Background}

During their sessile growth, plants need to deal with various environmental stresses caused by biotic and abiotic factors. In thousands of years' evolution, plants respond to these stresses through activation of series of responding molecules (Baniwal et al. 2004; Sunkar 2010). These defensing elements include TFs (Shi et al. 2018; Viana et al. 2018; Xiao et al. 2013), chaperone (Attallah et al. 2007), mitogen-activated protein kinase (Liang and Zhou, 2018), reactive oxygen species (ROS) (Miller et al. 2008), plant hormones (Bari and Jones, 2009; Peleg

\footnotetext{
* Correspondence: xxm@sippe.ac.cn

${ }^{1}$ Key Laboratory of Insect Developmental and Evolutionary Biology, Institute of Plant Physiology and Ecology, Shanghai Institutes for Biological Sciences, Chinese Academy of Sciences, Shanghai 200032, China

Full list of author information is available at the end of the article
}

and Blumwald, 2011; Santino et al. 2013) and even sugar (Wingler and Roitsch, 2008).

bHLH proteins are the largest TF family in plants that function extensively in plant development and defensive response through crosstalk among different signaling pathways (Ezer et al. 2017; Kazan and Manners, 2013). The bHLH protein Myelocytomatosis protein 2 (MYC2) is involved in JA-regulated plant development (Dombrecht et al. 2007), root formation (Chen et al. 2011), insect resistance (Schweizer et al. 2013) and pathogen response (Kazan and Manners, 2013), and is becoming a master regulator of JA-mediated responses. Rice OsbHLH148 is involved in JA signaling by interacting with OsJAZ and mediates drought tolerance (Seo et al. 2011). However, HLH proteins are a small group of atypical members in the bHLH family that lack the basic domain and usually form dimers or multimers with bHLH proteins and inhibit 
their transcriptional activity (Carretero-Paulet et al. 2010; $\mathrm{Li}$ et al. 2006). In rice, positive regulator of grain length 2 (PGL2) regulates grain weight by influencing cell elongation and interacting with a bHLH protein Antagonist of Pgl1 (APG) (Heang and Sassa, 2012). In Arabidopsis, HLH proteins ATBS1-interacting factors (AIFs) and ILI1 binding bHLH (IBH1) respectively interact with paclobutrazol resistance1 (PREs) and activators for cell elongation 1 (ACE1) to form HLH-bHLH complex and mediate brassinosteroid (BR) signaling and cell elongation (Wang et al. 2009), and this regulating pathway is conserved in rice (Zhang et al. 2009). A recent study in rice indicates that a HLH protein BR upregulated 1-like (OsBUL1) regulates cell elongation by forming an OsBUL1 complex1 (Jang et al. 2017). Nevertheless, it is not clear whether HLH proteins function in stress response.

The brown planthopper $(\mathrm{BPH})$ is a rice-specific herbivore, which causes severe yield losses each year in rice planting areas throughout Asia (Cheng et al. 2013; Flowers 2004). JA and SA mediated signaling pathways have been extensively identified in plant stress response against pathogen and insect (Berens et al. 2017). Generally, SA is proved to positively regulate rice defense to BPH (Yang and Zhang, 2016). Although the role of JA in $\mathrm{BPH}$ resistance is still controversy (Yang and Zhang, 2016), increasing evidence supports the negative role of $\mathrm{JA}$ in BPH resistance. For example, silencing of herbivore-induced rice type 2 13-lipoxygenase (OsHI-$L O X$ ) in JA synthesis is resistant to BPH (Zhou et al. 2009), and gain of function of 9-lipoxygenase gene (Osr9-LOX1) is favorable for the survival of the BPH larva (Zhou et al. 2014). The resistance genes Bph14 and Bph29 could both increase expression of genes in SA pathway and suppress genes in the JA pathway (Du et al. 2009; Wang et al. 2015). However, Bph6 and Bph9 could simultaneously activate SA and JA signaling pathway (Zhao et al. 2016; Guo et al. 2018), indicating that the specific role of JA in different background might also influence by the resistance genes. Nevertheless, the crosstalk between JA and SA in mediating BPH resistance is still unclear.

$P R$ genes are key factors in the immune pathway and function as markers for systemic acquired resistance (SAR) response (Glazebrook 2005). Expression of several $P R 1$ genes is significantly induced by rice blast (Mitsuhara et al. 2008); and transgenic tobacco plants expressing high level of PR-la protein are resistant to pathogens (Alexander et al. 1993). Some PR1 proteins are proved to be antifungal (Niderman et al. 1995). Expression of some $P R$ genes in SA signaling transduction is greatly influenced by the >non-expressor of pathogenesis-related genes $1(O s N P R 1)$ in mediating resistance to bacterial and rice blast (Sugano et al. 2010). Arabidopsis monomer NPR1 can enter into the nucleus and interact with TGACG motif-binding factor (TGA), which directly regulates the transcription of some $P R$ genes (Chern et al. 2014; Despres et al. 2000; Zhang et al. 2003). In rice, rTGA2.2 could interact with OsNPR1 (Chern et al. 2005), and rTGA 2.1 negatively regulates bacterial diseases and PR10 gene (Fitzgerald et al. 2005). Transgenic rice lines carrying the OsAOS 2 gene under the control of a strong, pathogen-inducible promoter exhibit enhanced activation of some $P R$ genes such as $P R 1 a, P R 3$, and $P R 5$, and increase resistance to rice blast (Mei et al. 2006). Also, some $P R$ genes could be induced by BPH (Hu et al. 2017).

Previously, we revealed that over-expression of allene oxide cyclase $(A O C)$ gene increased resistance to $\mathrm{BPH}$ in a JA-independent manner (Guo et al. 2014). To further reveal the downstream genes in $A O C$-mediated $\mathrm{BPH}$ resistance, we identified OsHLH61, which was upregulated in $A O C$ over-expressing plants. Function analysis revealed that OsHLH61 positively regulated $\mathrm{BPH}$ resistance by influencing expression of $P R 1, P R 5$ and PR10 genes. OsbHLH96 was proved to be the interacting factor of OsHLH61, and OsJAZ3 might interact with OsbHLH96. Meanwhile, OsbHLH96 could regulate the expression of $P R$ genes negatively. Therefore, we revealed the role of OsHLH61-OsbHLH96 complex in defense to $\mathrm{BPH}$ and added new points in SA and JA crosstalk in defending against $\mathrm{BPH}$.

\section{Results}

\section{Homologous comparison and phylogenetic analysis of the OsHLH61 sequence}

We identified OsHLH61 (Os07g0676600) gene in AOC over-expressing plants (Guo et al. 2014), and then cloned it. The coding region of OsHLH61 gene contains 474 base pairs. The encoded protein OsHLH61 contains an atypical HLH domain in the 2-50 amino acids and belongs to group D (Atchley and Fitch, 1997), which lacks the basic domain, and so that could not directly bind to DNA (Li et al. 2006). Furthermore, members in group D could negatively regulate the bHLH proteins and repress their transcriptional activation (Sun et al. 1991). To further analyze OsHLH61, we selected some homologous genes in monocotyledonous plants Oryza brachyantha and Zea mays, and dicotyledonous plants Cajanuy caja, Theobroma cacao and Gossyplum arboretum, and performed sequence alignment. It was revealed that the HLH domains were highly conserved; and the $\alpha$-helix in the HLH domain each had two conserved sites, Leu12 and Leu44 (Fig. 1a), which have been proved to be pivotal for dimerization (Brownlie et al. 1997; Carretero-Paulet et al. 2010). analysis using Neighbor-Joining, and revealed that OsHLH61 is nearest to the ObSCREAM2-like protein in wild rice (Fig. 1b). 
a

Consensus

1. OSHLH61

2. ObSCREAM2-like

3. ZmHLH61-like

4. CcbHLH61

5. GabHLH61-like

6. TcbHLH61

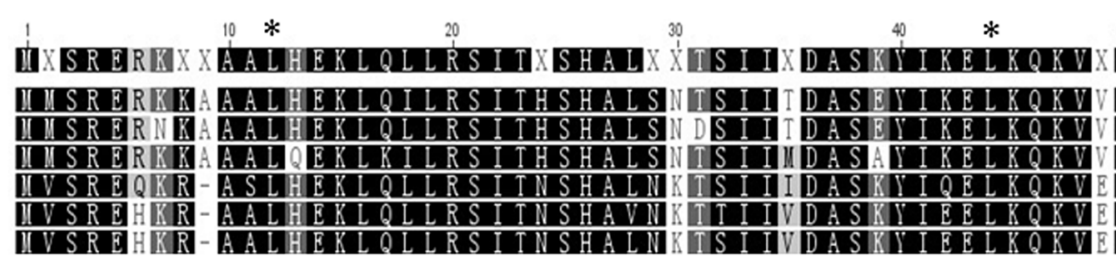

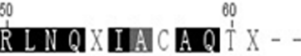

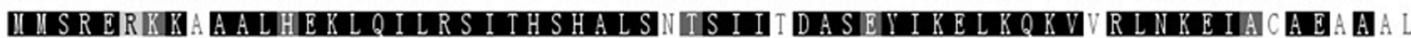

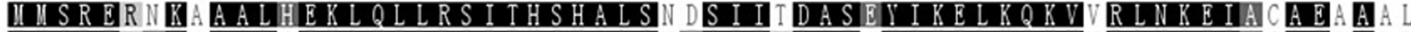

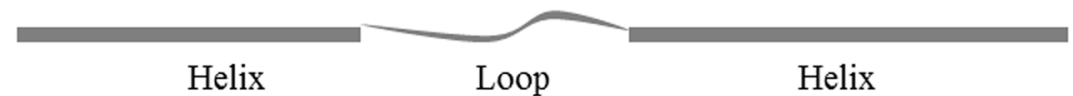

Helix Hoop Helix

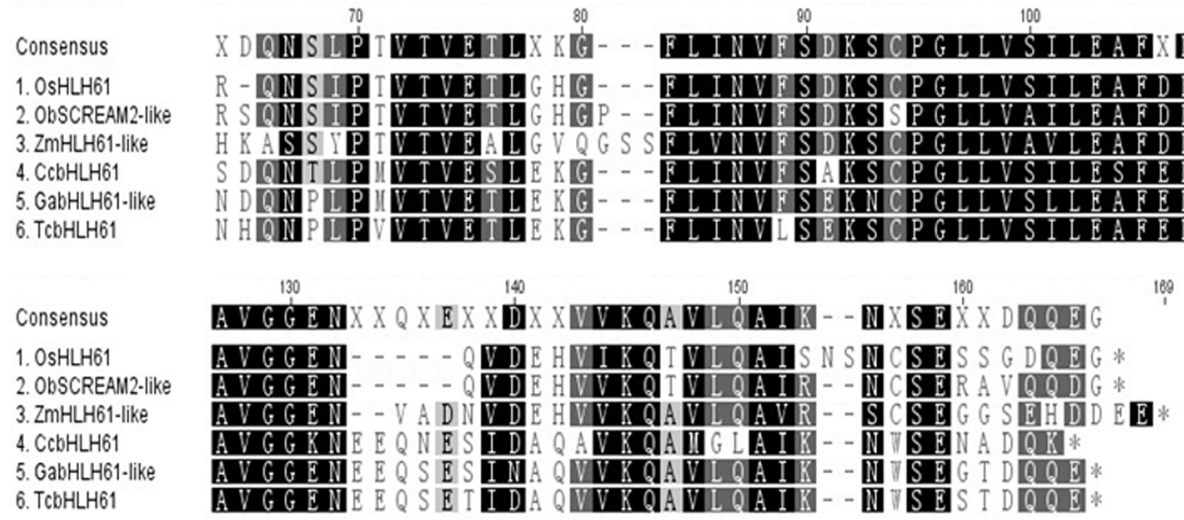

b

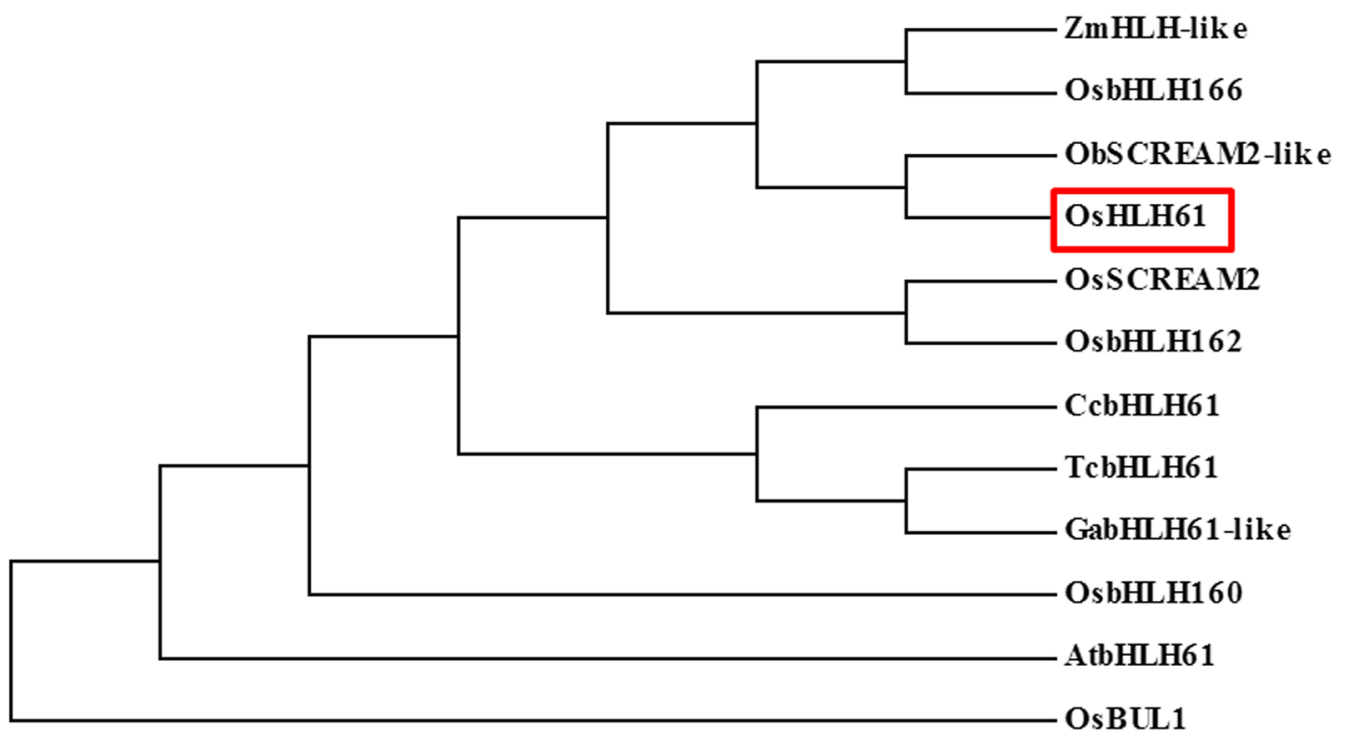

Fig. 1 Sequence alignment and phylogenetic tree of the homologues genes of OsHLH61. a Sequence alignment of six HLH61 proteins from different species, the helix-loop-helix region was sketched, and asterisks indicated the conserved Leu12 and Leu44 positions. $\mathbf{b}$ Phylogenic tree of OsHLH61 and its homologues using the neighbor-joining method with 500 replications in Mega 6.0. OsHLH61 was indicated by red rectangular

Expression profile of OsHLH61 and subcellular localization of OsHLH61 protein

To verify the response of OsHLH61 to BPH, we checked expression of OsHLH61 using a quantitative reverse transcriptase PCR (qRT-PCR) after BPH infestation, it was revealed that OsHLH61 was induced as early as $3 \mathrm{~h}$, and reached a peak at $7 \mathrm{~h}$ (Fig. 2a). So that OsHLH61 can be induced by BPH infestation.
Next, we checked the expression of OsHLH61 in different tissues, it was revealed that OsHLH61 expressed highly in the leaf sheath (Fig. 2b), where BPH feeds. Furthermore, we constructed an OsHLH61p::GUS plants to monitor the tissues where OsHLH61 proteins expressed. In consistence, GUS signal was concentrated in the leaf sheath (Fig. 2c). Besides, GUS signal was also detected in the young stem, and the initiation sites of root hair (Fig. 2c). 


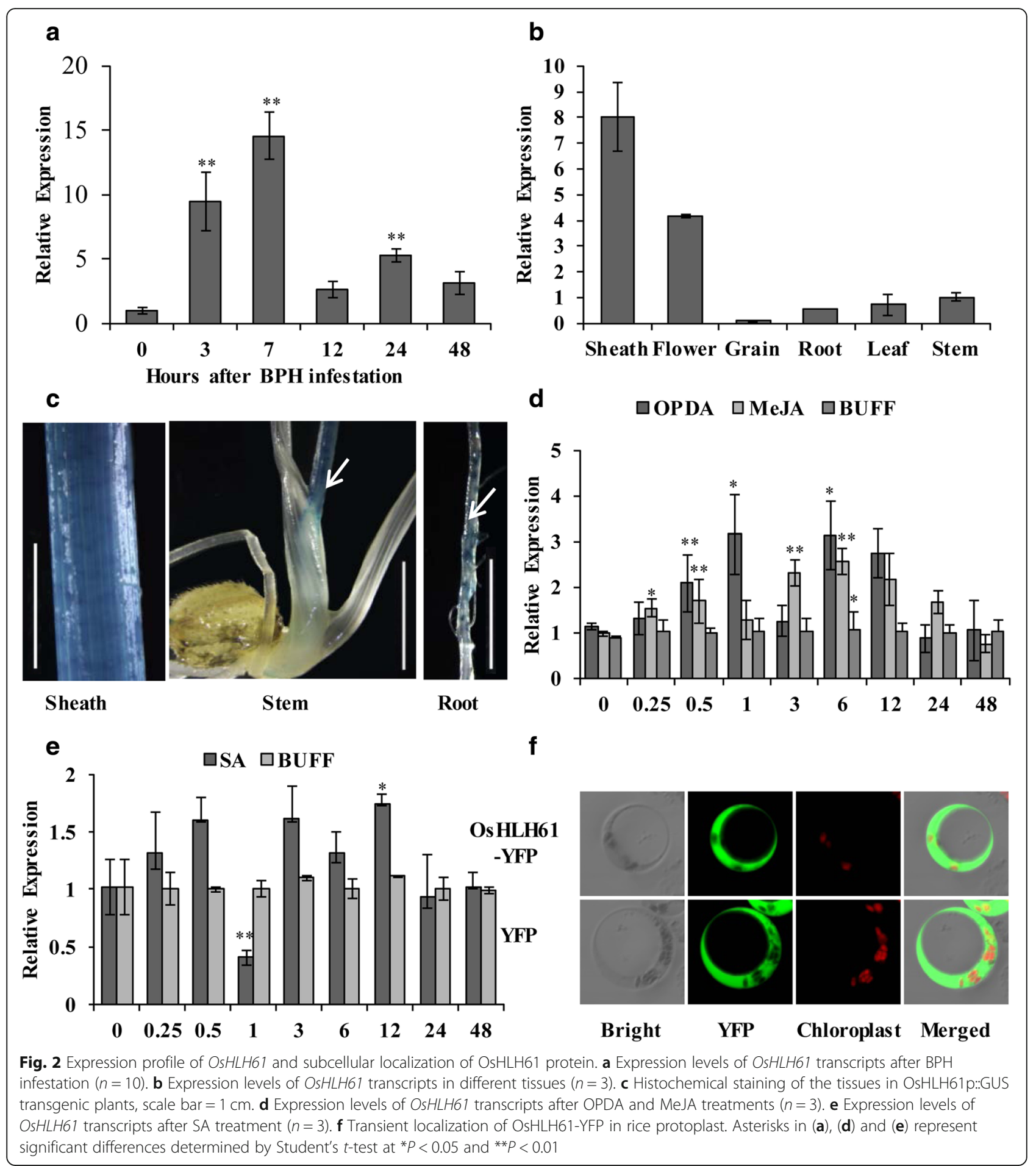

Since expression of OsHLH61 was influenced by AOC, which function in JA and OPDA biosynthesis (Guo et al. 2014), we further checked if OsHLH61 was responsive to MeJA and OPDA, it was revealed that OsHLH61 could be induced by both MeJA and OPDA (Fig. 2d). Meanwhile, under SA treatment, OsHLH61 was down-regulated at $1 \mathrm{~h}$ (Fig. 2e).
To further study the molecular basis of the OsHLH61 function, we checked the subcellular localization of the OsHLH61 protein. OsHLH61 localized ubiquitously in the protoplast, with the exception to the chloroplast (Fig. 2f), this was in consistence with that of OsBUL1 in rice (Jang et al. 2017). So that, HLH protein might be ubiquitously localized, different from bHLH protein, 
a
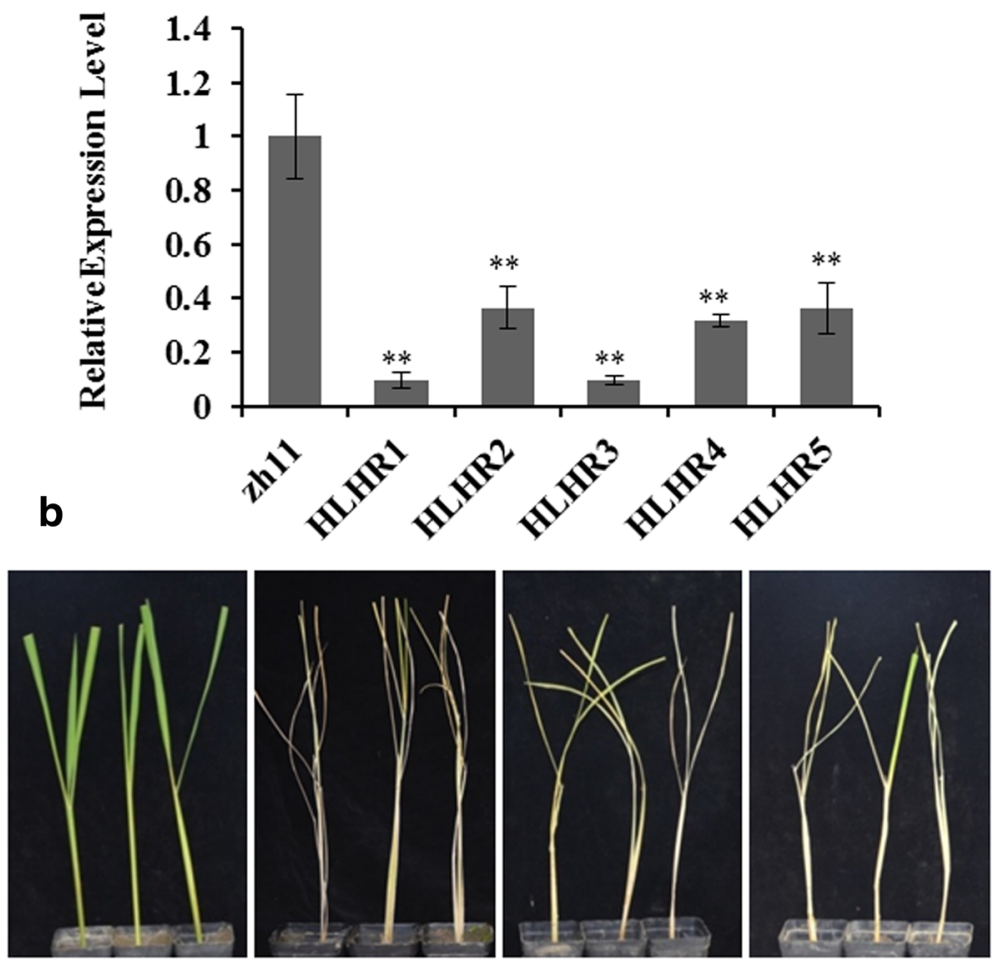

ZH11

HLHR2-8

HLHR4-11

HLHR5-2

C

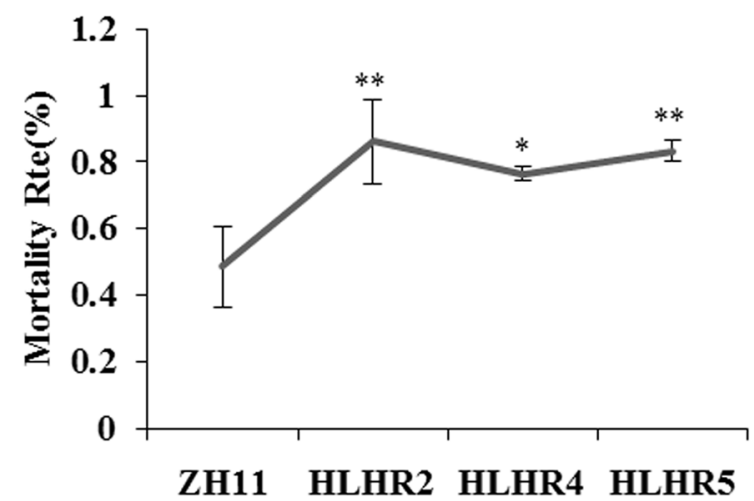

Fig. 3 Functional characterization of OsHLH61. a Expression levels of OsHLH61 transcripts in HLHR lines and WT $(n=3)$. $\mathbf{b}$ Phenotypes of HLHR lines after BPH infestation. c Statistic analysis of the survival rates of HLHR lines after BPH infestation $(n=30)$. Asterisks in (a) and (c) represent significant differences determined by Student's $t$-test at ${ }^{*} P<0.05$ and ${ }^{* *} P<0.01$

which are localized in the nucleus (Cui et al. 2016; $\mathrm{Xu}$ et al. 2014).

Knockdown of OsHLH61 rendered the plant more susceptible to BPH

To investigate the genetic function of OsHLH61, we constructed RNAi plants (HLHR) of OsHLH61 by transforming the RNAi plasmid into wild type (WT) ZH11. We chose 5 lines with obvious down-regulation of OsHLH61 (Fig. 3a) for further analysis. Expression of HLH61-like (OsbHLH166,
Os03g0338400), the homologous gene of OsHLH61 in rice, was not influenced (Additional file 1: Figure S1a), indicating the specific down-regulation of OsHLH61 in these HLHR plants. Then we performed individual analysis and revealed that the HLHR plants were more susceptible to $\mathrm{BPH}$ infestation (Fig. 3b). In consistence, the mortality of the HLHR plants was much higher than the WT after BPH infestation (Fig. 3c).

Besides, knock down of OsHLH61 influenced rice development. The leaves of the HLHR plants curled 
adaxially (Additional file 1: Figure S1b) and the tiller number increased (Additional file 1: Figure S1c). In addition, the fertility of the HLHR plants was reduced (Additional file 1: Figure S1 d, e).

\section{OsHLH61 interacted with OsbHLH96}

Since OsHLH61 was an atypical bHLH protein that needs to form HLH-bHLH complex in functioning, we tried to search the possible interacting bHLH protein of OsHLH61. There are 177 bHLH proetins in rice, with 26 atypical HLHs and 151 typical bHLHs (Carretero-Paulet et al. 2010; Li et al. 2006). We screened a yeast library and identified one bHLH protein, OsbHLH96, to interact with OsHLH61 in yeast two-hybrid analysis (Fig. 4a). BILC assay verified the interaction between OsHLH61 and OsbHLH96 (Fig. 4b). We found that OsHLH61 could not form homodimers in yeast two-hybrid test (Fig. 4a), but OsbHLH96 could (Fig. 4c). So that OsbHLH96 is the interacting protein of OsHLH61.

It is reported that AtMYC2 (Thireault et al. 2015) and OsbHLH148 (Seo et al., 2011) can respectively interact with JAZs in functioning. We wondered if OsbHLH96 could interact with OsJAZs, so that we checked OsJAZ1, OsJAZ3, OsJAZ5, OsJAZ7, OsJAZ9, OsJAZ11 and OsJAZ12, and revealed that only OsJAZ3 could interact with OsbHLH96 in yeast two-hybrid analysis (Fig. 4c).

\section{Some $P R$ s were significantly down-regulated in OsHLH61 RNAi plants}

To analyze the genes involved in OsHLH61 functioning, we carried out a RNA-seq analysis of the HLHR-4 plants before and after $\mathrm{BPH}$ infestation. Three kind of samples, WT ZH11, HLHR-4 without BPH infestation, and HLHR-4 after BPH infestation for $12 \mathrm{~h}$ (named as $\mathrm{ZHO}, \mathrm{HRO}$ and HR12 respectively), were used for analysis. We found that most $P R$ genes were down-regulated in HR0 compared with in $\mathrm{ZHO}$, so that data of $P R$ genes were extracted and displayed in heat map (Fig. 5a), it was revealed that most $P R$ genes in HR0 were down-regulated. Because the expression of PR1a (Os07g0129200), PR5 (Os04g0689900) and PR10a (Os12g0555500) were influenced by BPH feeding (Hu et al. 2017), we further checked the expression of PR1a, PR5, PR10a, PR1-like (PR1L, Os07g0127500) and some PR1 (Os07g0125201, Os07g0125000, Os07g0124900) by qRT-PCR. It was revealed that all of them were down-regulated in the HR0 plants (Fig. 5b). Besides, expression of the OsHLH61-like was not influenced in RNA-seq analysis, further indicating that OsHLH61 was specifically down-regulated in the HLHR plants (data not shown).

\section{Over-expression of OsbHLH96 down-regulated expression of some $P R$ genes}

Now that OsbHLH96 is the interacting protein of OsHLH61, we want to know if OsbHLH96 could regulate expression of $P R$ genes. We constructed the over-expression lines of OsbHLH96 (bHOE) and selected two positive lines for further analysis (Fig. 6a). Through qRT-PCR analysis, it was revealed that PR1a, PR1L, PR5 and PR10a genes were down-regulated in OsbHLH96 over-expressing lines (Fig. 6b). Now that expression of $P R$ genes was influenced by OsbHLH96, we further analyzed if there are

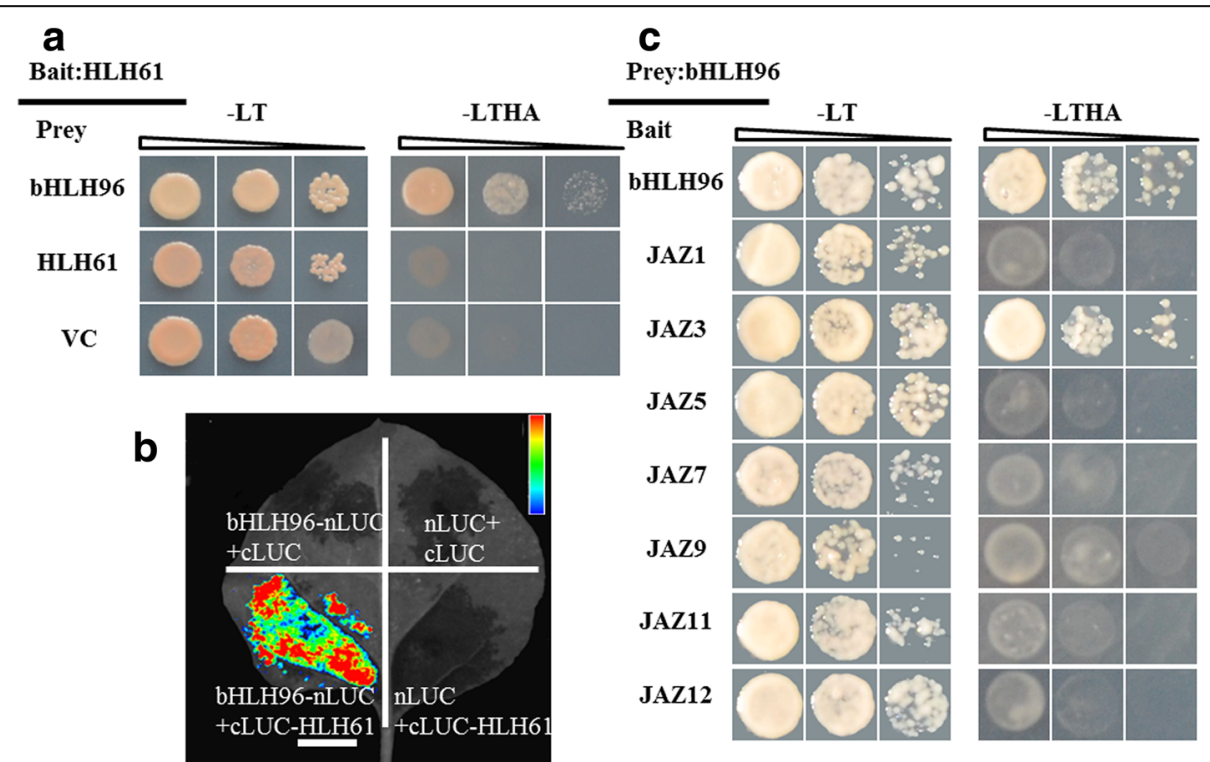

Fig. 4 Interaction analysis of OsHLH61 and OsbHLH96, and OsbHLH96 and OsJAZ3. a Yeast two-hybrid analysis of OsHLH61 and bHLH96; b BiLC assay of OsHLH61 and OsbHLH96 in tobacco. c Yeast two-hybrid analysis of OsJAZs and OsbHLH96. -LT, -LTHA indicated SD medium without Leu and Trp amino acids, and Leu, Trp, His and Ade amino acids respectively 


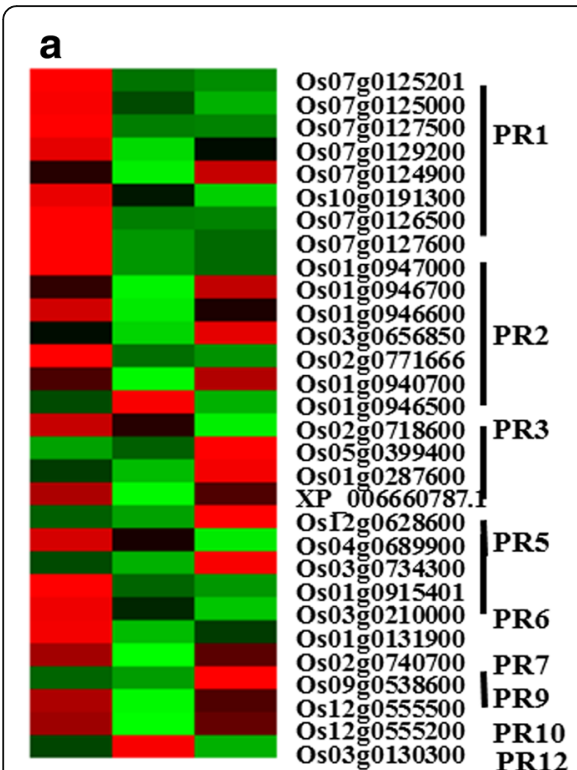

ZH0 HR0 HR12

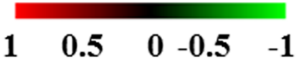

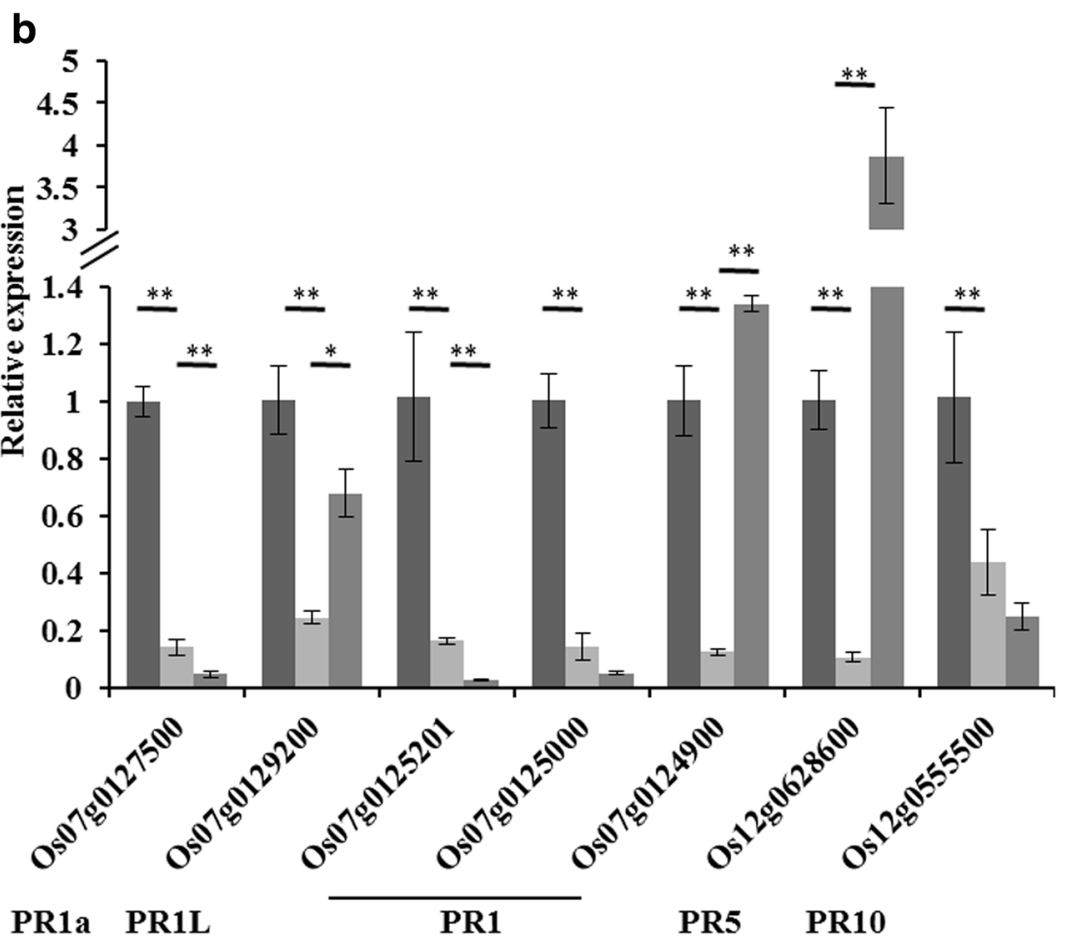

Fig. 5 Expression analysis of $P R$ genes in HLHR plants. a Expression levels of $P R$ transcripts in WT and HLHR plants revealed by the RNA-seq analysis. $\mathbf{b}$ qRT-PCR analysis of some PR genes in the HLHR-4 plants before and after BPH infestation, and in the WT $(n=3)$. Asterisks in (b) represent significant differences determined by Student's $t$-test at ${ }^{*} P<0.05$ and ${ }^{* *} P<0.01$ any E-box motifs for bHLH protein binding in the promoters of the $P R$ genes (Goossens et al. 2016). The promoters of PR1a, PR5, PR10a and AOS2 (Os03g0225900) have some E-box motifs (http://plantpan2.itps.ncku.edu.tw). Next, we performed Dual-Luc assay to determine whether OsbHLH96 could directly regulate $P R$ genes. Neither the luciferase signals (Fig. 6c) nor the LUC/RLUC ratios (Fig. 6d) support a direct regulation. $P R$ genes can be induced by SA (Agrawal et al., 2000), we chose PR1L to determine its expression under SA treatment. It was revealed that PR1L was induced by SA (Fig. 6e). However, OsbHLH96 was repressed by SA treatment (Fig. 6f).

\section{Discussion}

In this study, we revealed that down-regulation of OsHLH61 showed significant sensitivity to $\mathrm{BPH}$, and proved the function of $\mathrm{HLH}-\mathrm{bHLH}$ heterodimer in $\mathrm{BPH}$ response. We showed that OsHLH61 form bHLH-HLH heterodimer with OsbHLH96 (Fig. 4a, b). In both OsHLH61 RNAi and OsbHLH96 over-expressing plants, PR genes were down-regulated (Fig. 5a, b; Fig. 6b). PR1a gene can be induced by small brown planthopper indigestion (Hao et al. 2011), and PR1b has been reported to be induced by brown planthopper indigestion $(\mathrm{Hu}$ et al. 2017). Some PR1 proteins are proved to be antifungal (Niderman et al. 1995). Our study indicated that down regulation of PR genes in OsHLH61 RNAi plants might account for their sensitivity to BPH (Fig. 3b).

$P R$ genes are considered as markers for plant resistance (Glazebrook, 2005, Liu et al., 2018, Liu et al., 2013). Some $P R$ genes were down-regulated in OsHLH61 RNAi plants (Fig. 5). OsHLH61 was induced by JA, OPDA, but repressed by SA (Fig. 2d, e), and up-regulated in $A O C$ and OPR3 over-expressing lines (Guo et al., 2014), while the transcriptional level of $A O C$ or OPR3 was unchanged in OsHLH61 RNAi plants (data not shown), demonstrating that OsHLH61 located downstream of both AOC and OPR3 in JA synthetic pathway. OsbHLH96 was inhibited by SA (Fig. 6g), while $P R$ genes were induced by SA (Fig. 6f), together with the down-regulation of $P R$ genes in the OsbHLH96 over-expressing lines (Fig. 6b), indicating OsbHLH96 to be a negative regulator of SA signaling pathway in mediating resistance. In this study, we proved that OsHLH61 could interact with OsbHLH96 (Fig. 4a, b). And OsJAZ3, a JA pathway repressor (Chini et al., 2007), might interact with OsbHLH96 (Fig. 4d). Therefore, the OsHLH61-OsbHLH96 complex might mediate the crosstalk between SA and JA in regulating $\mathrm{BPH}$ resistance.

Furthermore, OsJAZ3 interacts with OsCOI1a (Os01g0853400) in the presence of $120 \mu \mathrm{M}$ coronatine (Seo et al., 2011). OsCOI1a-silenced plants are more sensitive to chewing insects (Ye et al., 2012), Meanwhile, 


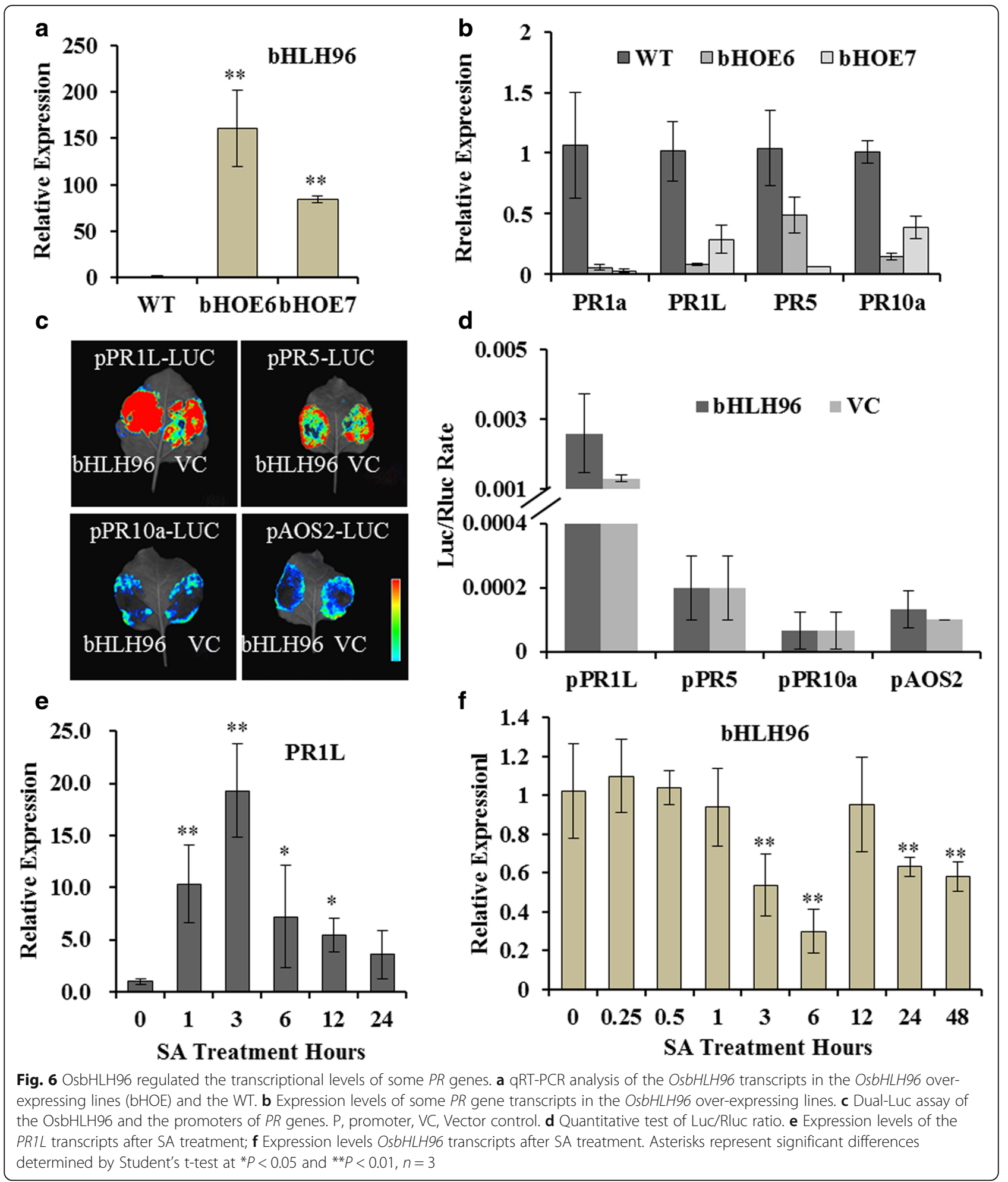

OsCOI1a influence the crosstalk between JA and GA (Yang et al., 2012). So that OsHLH61-OsbHLH96-OsJAZ3 might form multimers in stress response and plant hormone crosstalk.
Some TFs regulate $P R$ genes directly (Zhang et al., 2003; Chern et al., 2014). Although expression of some $P R$ genes were down-regulated in the OsHLH61 RNAi plants and the OsbHLH96 over-expressing lines (Fig. 5; Fig. 6f), direct 
regulation of OsbHLH96 to the $P R$ genes was not detected (Fig. 6c, d). There might be other TFs to regulate $P R$ genes instead. In the OsHLH61 RNAi plants, expressions of more than 30 TF-encoding genes were influenced (Additional file 2: Table S2). These TFs might be powerful candidates for direct regulation of $P R$ gene.

\section{Conclusion}

In this study, OsHLH61 RNAi plants were more susceptible to BPH than WT. OsHLH61 and OsbHLH96 can form heterodimer in functioning, and regulate the expression of $P R$ genes positively and negatively respectively, their antagonism in regulating $P R$ genes might be important to the understanding of the crosstalk between SA and JA signaling in mediating $\mathrm{BPH}$ resistance.

\section{Materials and methods}

\section{Plant and insect}

The WT rice variety is ZH11 (Oryza sativa L. subsp. japonica cv. Zhonghua No.11). Rice plants were grown in a greenhouse at $28 \pm 2{ }^{\circ} \mathrm{C}$ with a 12-h light/12-h dark cycle, and $70 \%-80 \%$ relative humidity. The $\mathrm{BPH}$ population was originally obtained from rice fields in Songjiang, Shanghai, China, and maintained on plants in a climate-controlled room at $26 \pm 2{ }^{\circ} \mathrm{C}, 12$-h light $/ 12$-h dark cycle and $80 \%$ relative humidity, or in the field under natural condition.

\section{BPH performance measurements}

Individual plant test was carried out at seedling stage using at least six replicates of each cultivar or line as previously described (Wang et al. 2012; Zhao et al. 2016). Each seedling about 3-week-stage was put under a plastic cage (diameter $4 \mathrm{~cm}$, height $8 \mathrm{~cm}$, with a breather window) infested with 12-18 s-instar BPH nymphs. Plant damage levels were observed daily, and 6-9 days later, the plants were scored as susceptible (dead) or resistant (alive).

\section{Constructs}

To construct the OsHLH61-RNAi plasmid, a 273-bp gene-specific fragment of the OsHLH61 coding sequence was amplified and cloned into PTCK303 vector in sense orientation by BamHI and KpnI, and in antisense orientation by SacI and SpeI.

To construct OsbHLH96 over-expression plasmid, the full-length OsbHLH96 was cloned into the BamHI and KpnI sites of pCambia1301-35SNOS vector, and got bHLH96OE plasmid.

Constructs used for rice protoplast transfection was generated with pA7-YFP, OsHLH61 cDNA sequence was cloned into the vector with pA7-HLH61-YFPF and.

pA7-HLH61-YFPR primers. For construction of OsHLH61p::GUS plasmid, a $2.27 \mathrm{~kb}$ promoter of OsHLH61 was cloned into p1300GUSNOS vector.
All the plasmids for genetic transformation were transformed into ZH11 using Agrobacterium-mediated method (Hiei et al., 1994). GUS activities were histochemically detected as described (Jefferson 1989).

For yeast two-hybrid analysis, the coding sequences of OsHLH61 and OsbHLH96 were cloned into pGADT7 and pGBKT7 vectors, the coding sequences of OsJAZ1, OsJAZ3, OsJAZ5, OsJAZ7, OsJAZ9, OsJAZ11 and OsJAZ12 were cloned into the pGADT7 vector. For BiLC analysis, the coding sequences of OsbHLH96 and OsHLH61 were cloned in-frame into the KpnI and SalI sites of 771-nluc and cluc-772 vector respectively.

For Dual-Luc analysis, the $2 \mathrm{~kb}$ promotor of OsPR1a, PR1L, OsPR5, OsPR10a and OsAOS2 were cloned into the KpnI and BamHI sites of pGreenII vector. The bHLH96OE plasmid and these plasmids were used for Dual-Luc analysis analyses (see the following).

\section{Rice protoplast transformation}

Rice protoplast transformation was performed by using polyethylene glycol -mediated transfections as described (Zhang et al. 2011). The YFP fluorescence signals for each combination were detected using an inverted confocal microscope (Olympus FV1000) 16 h after incubation. YFP fluorescent and chlorophyll auto-fluorescent signals were imaged at $514 \mathrm{~nm}, 527-532 \mathrm{~nm}$ and $650-798 \mathrm{~nm}$ respectively.

\section{Plant treatments}

For BPH treatment, plants about 2-week-old were infested with second-instar BPH nymphs after starvation for $2 \mathrm{~h}$ at a rate of 5 insects per seedling, and stem were collected after $0,3,6,12,24$ and $48 \mathrm{~h}$. For phytohormone treatment, rice plants were sprayed with $4 \mathrm{~mL}$ of MeJA $(400 \mu \mathrm{M})$, OPDA $(100 \mu \mathrm{M})$, or SA $(500 \mu \mathrm{M})$ solution in Dimethyl sulfoxide (DMSO), which was sprayed as control. Plant samples at $0,0.5,1.5,3,6,12,24$ and $48 \mathrm{~h}$ were collected and stored at $-80^{\circ} \mathrm{C}$ before RNA extraction.

\section{Quantitative real-time PCR (qRT-PCR) analysis}

Stem of 14-day-old WT plants were used to examine transcript levels of target genes before/after plants were infested with BPH. Three independent biological samples were used. Total RNA was isolated by using Trizo (Thermo). $1 \mu \mathrm{g}$ of total RNA was reverse-transcribed using the First Strand cDNA Synthesis Kit (Toyobo), according to the manufacturer's protocol. The qRT-PCR was performed with the SYBR Green Real-time PCR Master Mix Kit (Toyobo). Ubiquitin (Os03g0131300) was used as an internal standard to normalize cDNA concentrations. 


\section{Data analysis}

Data differences in different lines or treatments were determined by analyzing variance followed by Student's $t$-test. All tests were carried out with GraphPad Prizm (https://www.graphpad.com/scientific-software/prism/).

\section{RNA-seq and analysis}

Seedlings of non-infected HLHR4 and ZH11 plants, and of BPH infected HLHR4 plants were collected and RNAs extracted. Library was constructed, and sequencing was performed on a BGISEQ-500 and analysis was carried out under the help of Beijing Genomic Institution (www.geno mics.org.cn, BGI, Shenzhen, China).

\section{Tree building}

A phylogenetic tree was constructed using MEGA 6.0 (http://www.megasoftware.net/index.html) and the NJ method with the following parameters, Poisson correction, pairwise deletion, and bootstrap (500 replicates; random seed).

\section{Yeast two hybrid screening}

The yeast two-hybrid screening was carried out as described by Clontech (https://www.takarabio.com/products/protein-research/two-hybrid-and-one-hybrid-systems/yeast-two-hybrid-system/matchmaker-gold-yeast-two-hybrid-system). The vector pGBKT7-OsHLH61 was transformed into AH109 instead of Y2HGOLD. The mated culture was plated on the medium lacking leucine, tryptophan, histidine and adenine QDO (SD-LTHA) agar plates, the culture medium plates were incubated for 3-5 $\mathrm{d}$, then put the colonies into new plates QDO for 3-5 d, yeast colony PCR were carried out and the PCR products sequenced.

Yeast two-hybrid assay was carried out using the lithium acetate/single-stranded carrier DNA/PEG method (Gietz and Schiestl, 2007).

\section{Bimolecular luciferase complementation (BiLC) assay and dual-luciferase (dual-Luc) reporter assay}

BiLC assay was carried out as described (Liu et al. 2018). OsbHLH96 was fused to nLUC, OsHLH61 was fused to cLUC. Both constructs were transformed into Agrobacterium strain GV3101. Overnight cultures were collected by centrifugation, re-suspended in MES buffer (10 mM

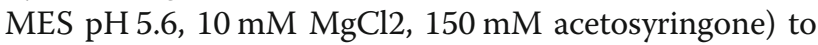
an OD600 of 1.0, mixed, nLUC and cLUC, bHLH96-nLUC and cLUC, nLUC and cLUC-HLH61 were used as control, and incubated at room temperature for $2-3 \mathrm{~h}$. The Agrobacteria suspension was drawn into a $10 \mathrm{~mL}$ syringe (without the needle) and press carefully by hand into healthy leaves of 3-week-old $N$. benthamiana plants. The plants were left under continuous white light for $2 \mathrm{~d}$ after infiltration. $1 \mathrm{mM}$ luciferin was infiltrated before the LUC signal was photographed with a cool CCD camera (Tanon 5200).

For Dual-Luc assay, OsbHLH96 and OsHLH61 were cloned into p1301-35SNOS vector, and the promotors of PR genes were cloned into pGreenII0800. Constructs for tests were then transformed into Agrobacterium strain GV3101 containing the pSoup-P19 plasmid (Hellens et al. 2005). LUC/RLUC ratio was measured using the Dual-Luciferase ${ }^{\oplus}$ Reporter Assay System kit (Promega).

The primer sequences used in this study were listed in Additional file 3: Table S2.

\section{Accession numbers}

Sequences in this study can be found in the National Center for Biotechnology Information (NCBI) OsHLH61 (Os07g0676600); OsbHLH96(Os03g0188400); OsJAZ3 (Os08g0428400); OsPR1a (Os07g0129200); PR1L (Os07g0127500); OsPR5 (Os04g0689900); OsPR10a (Os12g0555500); OsAOS2 (Os03g0225900); OsJAZ1 (Os04g0653000); OsJAZ5(Os04g0395800); OsJAZ7 (Os07g0615200); OsJAZ9(Os03g0180800);OsJAZ11 (Os03g0180900); OsJAZ12(Os10g0392400); Ubiquitin (Os03g0131300).

\section{Additional files}

\section{Additional file 1: Figure S1. Other phenotypes of the HLHR plants.} (DOCX $626 \mathrm{~kb}$ )

Additional file 2: Table S1. List of TF genes influenced by OsHLH61. (XLSX $11 \mathrm{~kb}$ )

Additional file 3: Table S2. Primer sequences used in this study. (XLSX $11 \mathrm{~kb})$

\section{Abbreviations}

OsAOS2: Allene Oxide Synthase 2; OsHLH61: Helix-loop-helix 61;

PRs: Pathogen-related genes; qRT-PCR: Quantitative real-time PCR

\section{Acknowledgements}

We are grateful to Dr. Hongtao Liu for providing the Dual-Luc system.

\section{Funding}

This work was supported by the National Transgenic Great Subject from the Ministry of Agriculture of China (2016ZX08009-003-001), the National Natural Science Foundation of China (31371949 and 31870232), and the grant from State Key Laboratory of Hybrid Rice (KF201805).

Availability of data and materials

All data supporting the conclusions of this article are provided within the article (and its additional files).

\section{Authors' contributions}

MW, DY, FM and MZ performed the experiments, analyzed the data. MW wrote the manuscript. ZS and XM revised the manuscript. All authors read and approved the manuscript.

Ethics approval and consent to participate Not applicable.

Consent for publication

Not applicable. 


\section{Competing interests}

The authors declare that they have no competing interests.

\section{Publisher's Note}

Springer Nature remains neutral with regard to jurisdictional claims in published maps and institutional affiliations.

\section{Author details}

${ }^{1}$ Key Laboratory of Insect Developmental and Evolutionary Biology, Institute of Plant Physiology and Ecology, Shanghai Institutes for Biological Sciences, Chinese Academy of Sciences, Shanghai 200032, China. ${ }^{2}$ University of Chinese Academy of Sciences, Beijing, China. ${ }^{3}$ Shanghai Chenshan Plant Science Research Center, Chinese Academy of Sciences/Shanghai Chenshan Botanical Garden, Shanghai 201602, China.

\section{Received: 9 September 2018 Accepted: 8 February 2019}

\section{Published online: 22 February 2019}

\section{References}

Agrawal G, Jwa N, Rakwal R (2000) A novel rice (Oryza sativa L.) acidic PR1 gene highly responsive to cut, phytohormones, and protein phosphatase inhibitors. Biochem Biophys Res Commun 274(1):157-165

Alexander D, Goodman R, Gut-Rella M, Glascock C, Weymann K, Friedrich L, Maddox D, Ahl-Goy P, Luntz T, Ward E (1993) Increased tolerance to two oomycete pathogens in transgenic tobacco expressing pathogenesis-related protein 1a. Proc Natl Acad Sci U S A 90:7327-7331

Atchley W, Fitch W (1997) A natural classification of the basic helix-loop-helix class of transcription factors. Proc Natl Acad Sci U S A 94:5172-5176

Attallah C, Welchen E, Gonzalez D (2007) The promoters of Arabidopsis thaliana genes AtCOX17-1 and-2, encoding a copper chaperone involved in cytochrome c oxidase biogenesis, are preferentially active in roots and anthers and induced by biotic and abiotic stress. Physiol Plant 129:123-134

Baniwal S, Bharti K, Chan K, Fauth M, Ganguli A, Kotak S, Mishra S, Nover L, Port M, Scharf K (2004) Heat stress response in plants: a complex game with chaperones and more than twenty heat stress transcription factors. J Biosci 29:471-487

Bari R, Jones J (2009) Role of plant hormones in plant defence responses. Plant Mol Biol 69:473-488

Berens M, Berry H, Mine A, Argueso C, Tsuda K (2017) Evolution of hormone signaling networks in plant defense. Annu Rev Phytopathol 55:401-425

Brownlie P, Ceska T, Lamers M, Romier C, Stier G, Teo H, Suck D (1997) The crystal structure of an intact human max-DNA complex: new insights into mechanisms of transcriptional control. Structure 5:509-520

Carretero-Paulet L, Galstyan A, Roig-Villanova I, Martinez-Garcia J, Bilbao-Castro J, Robertson D (2010) Genome-wide classification and evolutionary analysis of the bHLH family of transcription factors in Arabidopsis, poplar, rice, moss, and algae. Plant Physiol 153:1398-1412

Chen Q, Sun J, Zhai Q, Zhou W, Qi L, Xu L, Wang B, Chen R, Jiang H, Qi J, Li X, Palme K, Li C (2011) The basic helix-loop-helix transcription factor MYC2 directly represses PLETHORA expression during jasmonate-mediated modulation of the root stem cell niche in Arabidopsis. Plant Cell 23:3335-3352

Cheng X, Zhu L, He G (2013) Towards understanding of molecular interactions between rice and the brown planthopper. Mol Plant 6:621-634

Chern M, Bai W, Ruan D, Oh T, Chen X, Ronald P (2014) Interaction specificity and coexpression of rice NPR1 homologs 1 and 3 (NH1 and NH3), TGA transcription factors and negative regulator of resistance (NRR) proteins. BMC Genomics 15:461

Chern M, Fitzgerald H, Canlas P, Navarre D, Ronald P (2005) Overexpression of a rice NPR1 homolog leads to constitutive activation of defense response and hypersensitivity to light. Mol Plant-Microbe Interact 18:511-520

Chini A, Fonseca S, Fernandez G, Adie B, Chico J, Lorenzo G-CG, Lopez-Vidriero I, Lozano F, Ponce M, Micol J, Solano R (2007) The JAZ family of repressors is the missing link in jasmonate signalling. Nature 448(7154):666-671

Cui J, You C, Zhu E, Huang Q, Ma H, Chang F (2016) Feedback regulation of DYT1 by interactions with downstream bHLH factors promotes DYT1 nuclear localization and anther development. Plant Cell 28:1078-1093

Despres C, DeLong C, Glaze S, Liu E, Fobert PR (2000) The Arabidopsis NPR1/ NIM1 protein enhances the DNA binding activity of a subgroup of the TGA family of bZIP transcription factors. Plant Cell 12:279-290
Dombrecht B, Xue G, Sprague S, Kirkegaard J, Ross J, Reid J, Fitt G, Sewelam N, Schenk P, Manners J, Kazan K (2007) MYC2 differentially modulates diverse jasmonate-dependent functions in Arabidopsis. Plant Cell 19:2225-2245

Du B, Zhang W, Liu B, Hu J, Wei Z, Shi Z, He R, Zhu L, Chen R, Han B, He G (2009) Identification and characterization of Bph14, a gene conferring resistance to brown planthopper in rice. Proc Natl Acad Sci U S A 106:22163-22168

Ezer D, Shepherd S, Brestovitsky A, Dickinson P, Cortijo S, Charoensawan V, Box M, Biswas S, Jaeger K, Wigge P (2017) The G-Box transcriptional regulatory code in Arabidopsis. Plant Physiol 175:628-640

Fitzgerald H, Canlas P, Chern M, Ronald P (2005) Alteration of TGA factor activity in rice results in enhanced tolerance to Xanthomonas oryzae pv. Oryzae. Plant J 43:335-347

Flowers T (2004) Improving crop salt tolerance. J Exp Bot 55:307-319

Gietz R, Schiestl R (2007) High-efficiency yeast transformation using the LiAc/SS carrier DNA/PEG method. Nat Protoc 2:31-34

Glazebrook J (2005) Contrasting mechanisms of defense against biotrophic and necrotrophic pathogens. Annu Rev Phytopathol 43:205-227

Goossens J, Mertens J, Goossens A (2016) Role and functioning of bHLH transcription factors in jasmonate signalling. J Exp Bot 68:1333-1347

Guo H, Li H, Zhou S, Xue H, Miao X (2014) Cis-12-oxo-phytodienoic acid stimulates rice defense response to a piercing-sucking insect. Mol Plant 7: 1683-1692

Guo J, Xu C, Wu D, Zhao Y, Qiu Y, Wang X, Ouyang Y, Cai B, Liu X, Jing S, Shangguan X, Wang H, Ma Y, Hu L, Wu Y, Shi S, Wang W, Zhu L, Xu X, Chen R, Feng Y, Du B and He G (2018) Bph6 encodes an exocyst-localized protein and confers broad resistance to planthoppers in rice. Nat Genet 50:297-306.

Hao Z, Wang L, He Y, Liang J, Tao R (2011) Expression of defense genes and activities of antioxidant enzymes in rice resistance to rice stripe virus and small brown planthopper. Plant Physiol Biochem 49:744-751

Heang D, Sassa H (2012) An atypical bHLH protein encoded by POSITIVE REGULATOR OF GRAIN LENGTH 2 is involved in controlling grain length and weight of rice through interaction with a typical bHLH protein APG. Breed Sci 62:133-141

Hellens R, Allan A, Friel E, Bolitho K, Grafton K, Templeton M, Karunairetnam S, Gleave A, Laing W (2005) Transient expression vectors for functional genomics, quantification of promoter activity and RNA silencing in plants. Plant Methods 1:13

Hiei Y, Ohta S, Komari T, Kumashiro T (1994) Efficient transformation of rice (Oryza sativa L.) mediated by agrobacterium and sequence analysis of the boundaries of the T-DNA. Plant J 6:271-282

Hu L, Wu Y, Wu D, Rao W, Guo J, Ma Y, Wang Z, Shangguan X, Wang H, Xu C, Huang J, Shi S, Chen R, Du B, Zhu L, He G (2017) The coiled-coil and nucleotide binding domains of BROWN PLANTHOPPER RESISTANCE14 function in signaling and RESISTANCE against Planthopper in Rice. Plant Cell 29:3157-3185

Jang S, An G, Li H (2017) Rice leaf angle and grain size are affected by the OsBUL1 transcriptional activator complex. Plant Physiol 173:688-702

Jefferson R (1989) The GUS reporter gene system. Nature 342:837-838

Kazan K, Manners J (2013) MYC2: the master in action. Mol Plant 6:686-703

Li X, Duan X, Jiang H, Sun Y, Tang Y, Yuan Z, Guo J, Liang W, Chen L, Yin J, Ma H, Wang J, Zhang D (2006) Genome-wide analysis of basic/helix-loop-helix transcription factor family in rice and Arabidopsis. Plant Physiol 141:1167-1184

Liang X, Zhou J (2018) Receptor-like cytoplasmic kinases: central players in plant receptor kinase-mediated signaling. Annu Rev Plant Biol 69:267-299

Liu W, Zhang F, Zhang W, Song L, Wu W, Chen Y (2013) Arabidopsis Di19 functions as a transcription factor and modulates PR1, PR2, and PR5 expression in response to drought stress. Mol Plant 6(5):1487-1502

Liu Y, Li X, Ma D, Chen Z, Wang JW, Liu H (2018) CIB1 and CO interact to mediate CRY2-dependent regulation of flowering. EMBO Rep 19:1-10

Mei C, Qi M, Sheng G, Yang Y (2006) Inducible overexpression of a rice allene oxide synthase gene increases the endogenous jasmonic acid level, PR gene expression, and host resistance to fungal infection. Mol Plant-Microbe Interact 19:1127-1137

Miller G, Shulaev V, Mittler R (2008) Reactive oxygen signaling and abiotic stress. Physiol Plantarum 133:481-489

Mitsuhara I, Iwai T, Seo S, Yanagawa Y, Kawahigasi H, Hirose S, Ohkawa Y, Ohashi $Y$ (2008) Characteristic expression of twelve rice PR1 family genes in response to pathogen infection, wounding, and defense-related signal compounds (121/180). Mol Genet Genomics 279:415-27.

Niderman T, Genetet I, Bruyere T, Gees R, Stintzi A, Legrand M, Fritig B, Mosinger E (1995) Pathogenesis-related PR-1 proteins are antifungal. Isolation and 
characterization of three 14-kilodalton proteins of tomato and of a basic PR-1 of tobacco with inhibitory activity against Phytophthora infestans. Plant Physiol 108:17-27

Peleg Z, Blumwald E (2011) Hormone balance and abiotic stress tolerance in crop plants. Curr Opin Plant Biol 14:290-295

Santino A, Taurino M, De Domenico S, Bonsegna S, Poltronieri P, Pastor V, Flors V (2013) Jasmonate signaling in plant development and defense response to multiple (a) biotic stresses. Plant Cell Rep 32:1085-1098

Schweizer F, Fernandez-Calvo P, Zander M, Diez-Diaz M, Fonseca S, Glauser G, Lewsey MG, Ecker JR, Solano R, Reymond P (2013) Arabidopsis basic helixloop-helix transcription factors MYC2, MYC3, and MYC4 regulate glucosinolate biosynthesis, insect performance, and feeding behavior. Plant Cell 25:3117-3132

Seo J, Joo J, Kim M, Kim Y, Nahm B, Song S, Cheong J, Lee J, Kim J, Choi Y (2011) OsbHLH148, a basic helix-loop-helix protein, interacts with OsJAZ proteins in a jasmonate signaling pathway leading to drought tolerance in rice. Plant J 65:907-921

Shi Y, Ding Y, Yang S (2018) Molecular regulation of CBF signaling in cold acclimation. Trends Plant Sci 23:623-637

Sugano S, Jiang C, Miyazawa S, Masumoto C, Yazawa K, Hayashi N, Shimono M, Nakayama A, Miyao M, Takatsuji H (2010) Role of OsNPR1 in rice defense program as revealed by genome-wide expression analysis. Plant Mol Biol 74:549-562

Sun X, Copeland N, Jenkins N, Baltimore D (1991) Id proteins Id1 and Id2 selectively inhibit DNA binding by one class of helix-loop-helix proteins. Mol Cell Biol 11:5603-5611

Sunkar R (2010) MicroRNAs with macro-effects on plant stress responses. Semin Cell Dev Biol 21:805-811

Thireault C, Shyu C, Yoshida Y, St Aubin B, Campos M, Howe G (2015) Repression of jasmonate signaling by a non-TIFY JAZ protein in Arabidopsis. Plant J 82(4):669-679

Viana VE, Busanello C, da Maia LC, Pegoraro C, Costa de Oliveira A (2018) Activation of rice WRKY transcription factors: an army of stress fighting soldiers?. Curr Opin Plant Biol 45:268-275.

Wang H, Zhu Y, Fujioka S, Asami T, Li J, Li J (2009) Regulation of Arabidopsis brassinosteroid signaling by atypical basic helix-loop-helix proteins. Plant Cell 21:3781-3791

Wang Y, Cao L, Zhang Y, Cao C, Liu F, Huang F, Qiu Y, Li R, Lou X (2015) Map-based cloning and characterization of BPH29, a B3 domain-containing recessive gene conferring brown planthopper resistance in rice. J Exp Bot 66:6035-6045

Wang Y, Li H, Si Y, Zhang H, Guo H, Miao X (2012) Microarray analysis of broadspectrum resistance derived from an indica cultivar Rathu Heenati. Planta 235:829-840

Wingler A, Roitsch T (2008) Metabolic regulation of leaf senescence: interactions of sugar signalling with biotic and abiotic stress responses. Plant Biol 10:50-62

Xiao J, Cheng H, Li X, Xiao J, Xu C, Wang S (2013) Rice WRKY13 regulates cross talk between abiotic and biotic stress signaling pathways by selective binding to different cis-elements. Plant Physiol 163:1868-1882

Xu F, Kapos P, Cheng Y, Li M, Zhang Y, Li X (2014) NLR-associating transcription factor bHLH84 and its paralogs function redundantly in plant immunity. PLoS Pathog 10:e1004312

Yang D, Yao J, Mei C, Tong X, Zeng L, Li Q, Xiao L, Sun T, Li J, Deng X, Lee C, Thomashow M, Yang Y, He Z, He S (2012) Plant hormone jasmonate prioritizes defense over growth by interfering with gibberellin signaling cascade. Proc Natl Acad Sci U S A 109(19):E1192-E1200

Yang L, Zhang W (2016) Genetic and biochemical mechanisms of rice resistance to planthopper. Plant Cell Rep 35(8):1559-1572

Ye M, Luo S, Xie J, Li Y, Xu T, Liu Y, Song Y, Zhu-Salzman K, Zeng R (2012) Silencing COl1 in rice increases susceptibility to chewing insects and impairs inducible defense. PLoS one 7(4):e36214

Zhang L, Bai M, Wu J, Zhu J, Wang H, Zhang Z, Wang W, Sun Y, Zhao J, Sun X, Yang H, Xu Y, Kim S, Fujioka S, Lin W, Chong K, Lu T, Wang Z (2009) Antagonistic $\mathrm{HLH} / \mathrm{bHLH}$ transcription factors mediate brassinosteroid regulation of cell elongation and plant development in rice and Arabidopsis. Plant Cell 21:3767-3780

Zhang Y, Su J, Duan S, Ao Y, Dai J, Liu J, Wang P, Li Y, Liu B, Feng D, Wang J, Wang $H$ (2011) A highly efficient rice green tissue protoplast system for transient gene expression and studying light/chloroplast-related processes. Plant Methods 7:30

Zhang Y, Tessaro M, Lassner M, Li X (2003) Knockout analysis of Arabidopsis transcription factors TGA2, TGA5, and TGA6 reveals their redundant and essential roles in systemic acquired resistance. Plant Cell 15:2647-2653
Zhao $Y$, Huang J, Wang Z, Jing S, Wang $Y$, Ouyang $Y$, Cai B, Xin X, Liu X, Zhang C, Pan Y, Ma R, Li Q, Jiang W, Zeng Y, Shangguan X, Wang H, Du B, Zhu L, Xu X, Feng Y, He S, Chen R, Zhang Q, He G (2016) Allelic diversity in an NLR gene $\mathrm{BPH} 9$ enables rice to combat planthopper variation. Proc Natl Acad Sci U S A 113:12850-12855

Zhou G, Qi J, Ren N, Cheng J, Erb M, Mao B, Lou Y (2009) Silencing OsHI-LOX makes rice more susceptible to chewing herbivores, but enhances resistance to a phloem feeder. Plant J 60:638-648

Zhou G, Ren N, Qi J, Lu J, Xiang C, Ju H, Cheng J, Lou Y (2014) The 9lipoxygenase Osr9-LOX1 interacts with the 13-lipoxygenase-mediated pathway to regulate resistance to chewing and piercing-sucking herbivores in rice. Physiol Plant 152:59-69

\section{Submit your manuscript to a SpringerOpen ${ }^{\odot}$ journal and benefit from:}

- Convenient online submission

- Rigorous peer review

- Open access: articles freely available online

- High visibility within the field

- Retaining the copyright to your article

Submit your next manuscript at $>$ springeropen.com 